\title{
Fulminant bilateral acute retinal necrosis syndrome associated with viral encephalitis: A case report
}

\author{
CHUNKUI ZHOU, LIJUN ZHU and SHAOKUAN FANG
}

Department of Neurology, The First Teaching Hospital of Jilin University, Changchun, Jilin 130041, P.R. China

Received June 22, 2015; Accepted July 29, 2016

DOI: $10.3892 / e t m .2016 .3594$

\begin{abstract}
Herpes simplex virus (HSV) is the most common cause of acute viral encephalitis. Acute retinal necrosis (ARN) is a rapidly progressing and potentially blinding eye disease that may be induced by HSV. The present case study reports the very rare case of a patient with herpes simplex encephalitis (HSE) combined with acute retinal necrosis (ARN). A 47-year-old woman was admitted to hospital with persistent high fever and somnolence for 5 days. Magnetic resonance imaging showed abnormal signals in the right medial temporal lobes, and HSV-1 was identified in the serum and cerebrospinal fluid. Five days later, despite treatment with intravenous acyclovir and partial improvement in consciousness, the patient suddenly developed blurred vision and bilateral visual pain. Fundus fluorescence angiography revealed bilateral vessel obstruction and flaky reduced fluorescence. ARN was diagnosed clinically. Dexamethasone was administered as an anti-inflammatory adjunct to intravenous acyclovir therapy. The visual acuity of the patient was reduced to mere light perception a further 4 days later. The present case indicates that HSE may be complicated with ARN, causing a reduction in visual acuity to mere light perception within a very short time.
\end{abstract}

\section{Introduction}

Herpes simplex encephalitis (HSE) is associated with high mortality and morbidity rates (1). Relapses and new infections at anatomically different sites, including the eye, may occur due to reactivation and neuronal translocation of the causative agent, herpes simplex virus (HSV) (2). HSE may be a risk factor for the development of acute retinal necrosis (ARN), a rapidly progressing and potentially blinding eye

Correspondence to: Professor Shaokuan Fang, Department of Neurology, The First Teaching Hospital of Jilin University, 71 Xinmin Street, Changchun, Jilin 130041, P.R. China E-mail: fang20063536@sina.com

Key words: herpes simplex encephalitis, acute retinal necrosis, fundus fluorescence angiography, brain magnetic resonance, herpes simplex virus disease induced by HSV (2). ARN was initially described as a rapidly progressive unilateral necrotizing retinitis in 1971 by Urayama et al (3). The prevalence of the disease is equal in both sexes and it typically occurs between the fifth and seventh decades of life (4). To prevent a poor outcome, ARN requires aggressive management (5). Although several case reports have described the occurrence of ARN after herpetic encephalitis (6), ARN following HSE is rare (7). In the present case report, the case of a healthy woman with bilateral ARN that occurred in combination with HSE following treatment with intravenous acyclovir is described.

\section{Case report}

A 47-year-old woman was admitted to the First Teaching Hospital of Jilin University (Changchun, China) in October 2013 with persistent high fever and somnolence for 5 days. Magnetic resonance imaging (MRI) showed abnormal signals in the right medial temporal lobes (Fig. 1). The history of the patient included frequent oral herpes lesions and infection of the upper respiratory tract. Therefore, viral encephalitis was suspected and treatment with intravenous acyclovir $(10 \mathrm{mg} / \mathrm{kg})$ was initiated and continued every $8 \mathrm{~h}$. HSV-1 was identified in the patient's serum and cerebrospinal fluid by polymerase chain reaction $(\mathrm{PCR})$, which was performed in our clinical laboratory. Examination of the cerebrospinal fluid also showed that the patient's glucose levels remained at a normal level ( $2.7 \mathrm{mmol} / \mathrm{l}$; normal range, $2.5-4.4 \mathrm{mmol} / \mathrm{l})$, while the monocyte count was elevated $\left(2.7 \times 10^{7} / 1\right.$; normal range, $\left.0-5 \times 10^{6} / 1\right)$. An electroencephalogram revealed intermittent rhythmic slowing in the bilateral hemispheres.

Five days after admission, the patient's consciousness was slightly improved, but she suddenly developed blurred vision and visual pain bilaterally. Dexamethasone $(15 \mathrm{mg} /$ day; Jilin Aodong Pharmaceutical Group Co., Ltd., Jilin, China) was administered intravenously for 15 days as an anti-inflammatory adjunct to intravenous acyclovir therapy. A further 4 days later, only light perception remained in both eyes. Examination of the left eye revealed extensive vitritis and retinal thickening with advanced retinal necrosis in the peripheral and central retina. The optic disc was swollen along with an inferior retinal detachment, extending to the macula. Extensive retinal vasculitis and hemorrhage were observed. Ultrasound examination showed a thickened temporal wall in the right eye (Fig. 2). Fundus fluorescence angiography (FFA) 


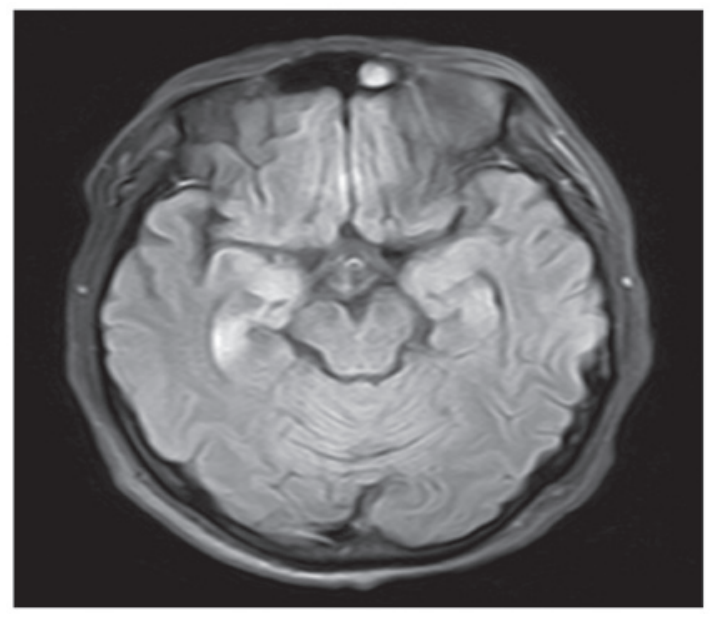

Figure 1. Brain magnetic resonance image showed abnormal signals in the right medial temporal lobes.

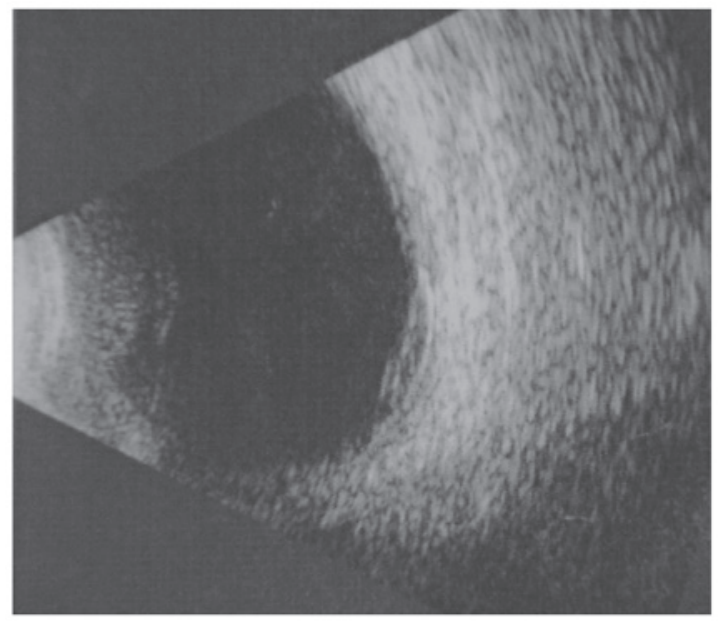

Figure 2. Ultrasound image revealed temporal wall thickening in the patient's right eye.

was conducted in the hospital ophthalmology department, and revealed bilateral vessel obstruction and a large amount of low fluorescence, with a flaky appearance, in the background (Fig. 3). HSE complicated with ARN was considered clinically, marked by weak light perception. HSV-1 was also detected through vitreous tap with PCR analysis and this confirmed the diagnosis. Following diagnosis, the patient was treated with intravenously administered acyclovir and dexamethasone, and mere light perception remained in each eye.

Informed consent was obtained from the patient for the publication of the present case study.

\section{Discussion}

The characteristics of ARN syndrome are anterior segment inflammation, along with periorbital pain, and a loss of vision due to vitreous opacification, necrotizing retinitis and, occasionally, optic neuropathy (8). In the past, ARN was only diagnosed clinically. However, as understanding of the pathophysiology of ARN advanced, adjunct laboratory tests were developed. According to the standard clinical criteria of the
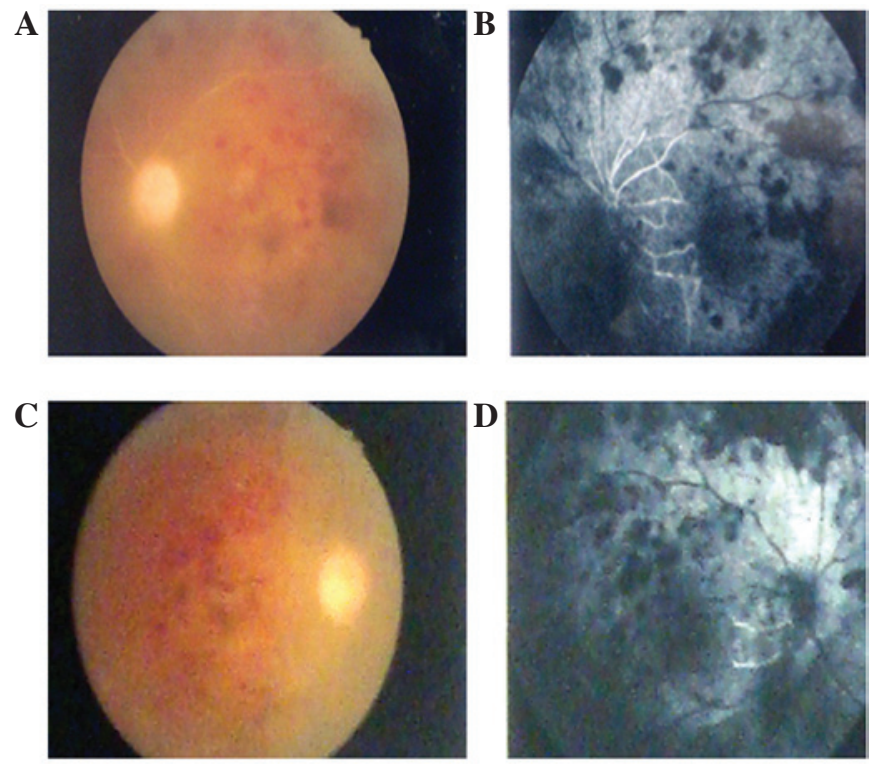

Figure 3. Fundus fluorescence angiography revealed bilateral vessel obstruction and flaky reduced fluorescence. (A) Fundus photograph (left eye). (B) Fluorescein angiogram (left eye). (C) Fundus photograph (right eye). (D) Fluorescein angiogram (right eye).

American Uveitis Society (9), the diagnosis of ARN can be made on the basis of focal, well-demarcated areas of retinal necrosis within the peripheral retina; rapid, circumferential progression of necrosis; and occlusive vasculopathy. PCR is a valuable diagnostic tool for the diagnosis of ARN (10). The patient described in the present case study manifested a classic case of fulminant bilateral ARN. In contrast to recovery from impaired light perception in reported cases of ARN syndrome $(11,12)$, the present patient responded poorly to antiviral and steroidal treatments. The poor visual outcome of this patient may be associated with impairment of the optic nerve in addition to extensive retinal detachment and her possible poor immune status.

HSV is the most common cause of acute viral encephalitis (13). The majority of infections in adults involve HSV-1, and few cases are attributed to HSV-2 (14). HSE typically affects the fronto-temporal lobe (1). The results of MRI and electroencephalography support the diagnosis of HSE in the present patient. The diagnosis of HSE complicated with ARN was supported by clinical findings and analysis of the cerebrospinal fluid and vitreous tap using PCR. The patient's cerebrospinal fluid and vitreous tap tested positive for HSV-1. Furthermore, the patient had a history of oral lesions. Although the majority of cases of HSV-related retinitis are established clinically, the patient's cerebrospinal fluid and vitreous tap tested positive for HSV-1. Several reports have described a retinitis that is consistent with ARN syndrome $(6,7,15)$. However, to the best of our knowledge there have not been any previous reports of fulminant HSE with ARN syndrome in which the visual ability was reduced to mere light perception within 4 days.

The potential of HSV to latently infect neurons within the central nervous system is well established. HSV is reactivated and spread via intra-axonal, trans-synaptic and extracellular mechanisms, resulting in damaging effects on remote and 
local tissue. The ability of HSV to migrate has been demonstrated in an animal model, where recombinant HSV was injected into the anterior chamber of one eye and retinitis subsequently occurred in the other eye, due to viral migration along the hypothalamus and contralateral optic nerve. It was hypothesized that the virus may have migrated from the suprachiasmatic nucleus to the retina by retrograde axonal transport along the optic nerve (16). Vandercam et al (17) described a case with an overall interval of 5 weeks (from 14 days to 2 months) between encephalitis and ARN. In the present patient, encephalitis occurred $\sim 1$ week prior to the symptoms of retinal disease. It is hypothesized that activated virus in the medial temporal lobes may have been transported to the retina axonally via the optic nerve, resulting in ARN.

\section{Acknowledgements}

The authors thank Dr. He for his excellent assistance in ophthalmology in the present study.

\section{References}

1. Whitley RJ: Herpes simplex virus infections of the central nervous system: A review. Am J Med 85: 61-67, 1988.

2. Ganatra JB, Chandler D, Santos C, Kuppermann B and Margolis TP: Viral causes of the acute retinal necrosis syndrome. Am J Ophthalmol 129: 166-172, 2000.

3. Urayama A, Yamad N, Sasaki T, et al. Unilateral acute uveitis with retinal periretinal arteritis and detachment. Jpn J clin Ophthalmol 25: 607-619, 1971.

4. Bodaghi B, Rozenberg F, Cassoux N, Fardeau C and LeHoang P: Nonnecrotizing herpetic retinopathies masquerading as severe posterior uveitis. Ophthalmology 110: 1737-1743, 2003.
5. Palay DA, Sternberg P Jr, Davis J, Lewis H, Holland GN, Mieler WF, Jabs DA and Drews C: Decrease in the risk of bilateral acute retinal necrosis by acyclovir therapy. Am J Ophthalmol 112: 250-255, 1991.

6. Gain P, Chiquet C, Thuret G, Drouet E and Antoine JC: Herpes simplex virus type 1 encephalitis associated with acute retinal necrosis syndrome in an immunocompetent patient. Acta Ophthalmol Scand 80: 546-549, 2002.

7. Tada Y, Negoro K, Morimatsu M, Makino H and Nishida T: Findings in a patient with herpes simplex viral meningitis associated with acute retinal necrosis syndrome. AJNR Am J Neuroradiol 22: 1300-1302, 2001.

8. Sergott RC, Belmont JB, Savino PJ, Fischer DH, Bosley TM and Schatz NJ: Optic nerve involvement in the acute retinal necrosis syndrome. Arch Ophthalmol 103: 1160-1162, 1985.

9. Holland GN; Executive Committee of the American Uveitis Society: Standard diagnostic criteria for the acute retinal necrosis syndrome. Am J Ophthalmol 117: 663-667, 1994.

10. Tran TH, Rozenberg F, Cassoux N, Rao NA, LeHoang P and Bodaghi B: Polymerase chain reaction analysis of aqueous humour samples in necrotizing retinitis. Br J Ophthalmol 87: 79-83, 2003.

11. Kianersi F, Masjedi A and Ghanbari H: Acute retinal necrosis after herpetic encephalitis. Case Rep Ophthalmol 1: 85-89, 2010.

12. Hirota K, Akimoto M and Katsura T: Bilateral acute retinal necrosis after herpetic meningitis. Clin Ophthalmol 6: 551-553, 2012.

13. Burk J and Miller JR: Infections of the nervous system. In: Merritt's Textbook of Neurology. Rowland LP (ed). Williams and Wilkins, Baltimore, MD, pp1546-159, 1995.

14. Whitley RJ: Herpes simples virus. In: Fields Virology. Fields BN, Knipe DM and Howley PM (eds). 3rd edition. Lippincott-Raven Publishers, Philadelphia, pp2323-2330, 1996.

15. Pepose JS, Kreiger AE, Tomiyasu U, Cancilla PA and Foos RY: Immunocytologic localization of herpes simplex type 1 viral antigens in herpetic retinitis and encephalitis in an adult. Ophthalmology 92: 160-166, 1985.

16. Vann VR and Atherton SS: Neural spread of herpes simplex virus after anterior chamber inoculation. Invest Ophthalmol Vis Sci 32: 2462-2472, 1991.

17. Vandercam T, Hintzen RQ, de Boer JH and Van der Lelij A: Herpetic encephalitis is a risk factor for acute retinal necrosis. Neurology 71: 1268-1274, 2008. 